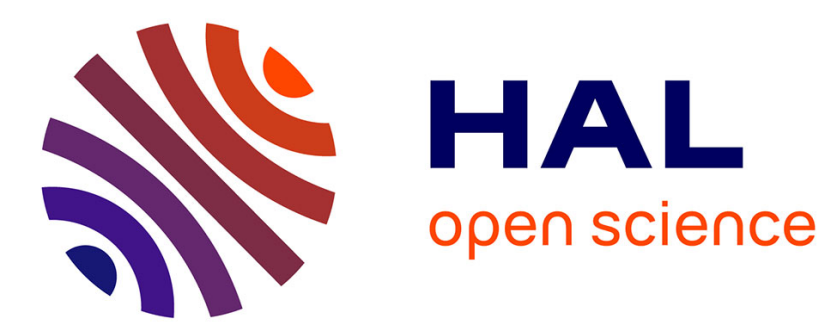

\title{
Robust sliding mode control of nonlinear systems with delay: A design via polytopic formulation
}

Frédéric Gouaisbaut, Yann Blanco, Jean-Pierre Richard

\section{To cite this version:}

Frédéric Gouaisbaut, Yann Blanco, Jean-Pierre Richard. Robust sliding mode control of nonlinear systems with delay: A design via polytopic formulation. International Journal of Control, 2004, 77 (2), pp.206-215. 10.1080/00207170310001633286 . inria-00266509

\section{HAL Id: inria-00266509 \\ https://inria.hal.science/inria-00266509}

Submitted on 23 Mar 2008

HAL is a multi-disciplinary open access archive for the deposit and dissemination of scientific research documents, whether they are published or not. The documents may come from teaching and research institutions in France or abroad, or from public or private research centers.
L'archive ouverte pluridisciplinaire HAL, est destinée au dépôt et à la diffusion de documents scientifiques de niveau recherche, publiés ou non, émanant des établissements d'enseignement et de recherche français ou étrangers, des laboratoires publics ou privés. 


\title{
Robust Sliding Mode Control of Nonlinear Systems with Delay: A Design via Polytopic Formulation.
}

\author{
F. Gouaisbaut, Y. Blanco, J.P. Richard \\ LAIL (CNRS UPRESA 8021), \\ Ecole Centrale de Lille, BP 48, \\ 59651 Villeneuve d'Ascq CEDEX - FRANCE. \\ e-mail : \{frederic.gouaisbaut,blanco, richard\}@ec-lille.fr \\ FAX: $(+33) 320335418$
}

keywords : nonlinear time delay systems, sliding mode control, LMIs.

\begin{abstract}
This paper outlines the possibility to control nonlinear uncertain systems with single, possibly timevarying state-delay. These systems are submitted to additive perturbations (possibly nonvanishing). The control is of sliding mode type and the sliding surface is designed to maximize the calculable set of delays which do not destabilize the relay-delay system. The way to deal with the nonlinear parts of the model relies on a polytopic formulation, i.e. a decomposition into a nonlinear combination of linear models, whose weighting coefficients satisfy a convex property. The conditions for the existence of the sliding regime are studied by using both a Lyapunov Krasovskii functionnal and a Lyapunov-Razumikhin function. The optimization procedure for the choice of the sliding surface is expressed in terms of LMIs. An example illustrates the proposed method.
\end{abstract}

\section{Introduction}

Control of time delay systems is a problem of present interest since the delay phenomemon is a natural aspect of the dynamic processes in many engineering fields (see for example [Gopalsamy, 1992]) and can induce instability or poor performances. For the last decade, several works have proposed criteria for the robust stabilization of time delay systems: for linear models with parameter uncertainties, see [Li \& Decarlo, 2003, Niculescu et al., 1998, Kolmanovskii et al., 1999, Nguang, 1998] (Lyapunov-Krasovski approach leading to LMIs) and [Dambrine et al., 1995] (comparison approach). The resulting control laws are of continuous, often memoryless, feedback type. The results concerning robustness with respect to external disturbances rely on either $H_{\infty}$ design (see [Li \& De Souza, 1996, Nguang, 1998] and references therein) or structural approaches, such as disturbance decoupling using models over rings (see [Conte \& Perdon, 1995] and references therein).

However, in the nonlinear case, a few approaches have been investigated. In [Goubet.-Bartholoméüs et al., 1997] the comparison method by vector norms is interesting for the stability problem but raises computationnal problems for the controller design. In [Sira-Ramirez \& Angulo-Nunez, 1998], the results of passivity-based control were extented to the case of nonlinear delay systems. Recently, approaches based on the input-ouput linearization have been used to asymptotically stabilize nonlinear time delay systems. Two main approaches have been proposed. 
First of all, [Moog et al., 2000] extended the structural appoach (i.e. ring models) to nonlinear models. In the other case (see [Germani et al., 1996]), the results are based on an extension of the Lie derivative approach to time delay systems. Nevertheless, both controllers, interesting in theory, are difficult to apply, because all of them assume that the delay is constant and known.

The Sliding Mode Control (SMC) approach [Utkin, 1991], based on the use of discontinuous control laws (relays), is known to be an efficient alternative way to tackle many challenging problems of robust stabilization. For instance, an appropriate sliding mode strategy can achieve stabilization by "dominating" nonlinear terms and additive disturbances, provided some appropriate "matching conditions" hold (roughly speaking, the disturbances must belong to the space spanned by the input function). However, the combination of delay phenomenon with relay actuators makes the situation much more complex (see the survey paper [Richard, 2003]) : designing a sliding controller without taking delays into account may lead to unstable or chaotic behaviors or, at least, results in highly chattering behaviors [Dambrine et al., 1998]. Even if the general framework of differential inclusions has been proposed in this case [Kolmanovskii \& Myshkis, 1999], the concrete control results are not so numerous

[Bonnet et al., 1998,

Choi, 1999, Gouaisbaut et al., 2001, Gouaisbaut et al., 1999, Koshkouei \& Zinober, 1996, Luo \& De la Sen, 1992, Shyu \& Yan, 1993]. In [Bonnet et al., 1998], the considered systems have output delay and relay actuators, but the study is limited to first order processes. In [Choi, 1999, Shyu \& Yan, 1993], the authors have considered linear model and constant delay.

The purpose of this paper is to extend the SMC approach of linear time delay systems to nonlinear ones. To this end, a polytopic formulation of the nonlinear systems is introduced [Takagi \& Sugeno, 1985, Tanaka et al., 1998]. The uncertainties are modelled as additive and possibly nonvanishing perturbations. Two kinds of delays are considered : firstly, constant and known, then varying and unknown.

The presentation is organized as follows: Section 2 is devoted to preliminary results (transformation of the original system into regular form) and formulation of the problem. Subsection 3.1 develops a sliding mode controller by means of an LMI approach when the delay is supposed to be constant. Then, in Subsection 3.2, we assume that the delay is time-varying and unknown. By a Razumikhin approach, a variable structure controller is investigated so to insure the asymptotic stability of the closed loop system. Section 4 gives an illustrative example.

\section{Notations}

In this paper, we consider the following nonlinear system with a possibly time varying delay $\tau=\tau(t) \geq$ 0 :

$$
\left\{\begin{array}{l}
\dot{x}(t)=f\left(x_{t}(\theta)\right)+g(x(t)) u(t)+p\left(x_{t}, t\right) \\
x(t)=\phi(t) \text { for } t \in[-\tau, 0]
\end{array}\right.
$$

The following notations are used:

$$
x \in \mathbb{R}^{n}, x_{t}(\theta)=x(t-\theta) \text { for } \theta \in[0, \tau(t)],
$$

$f$ is a non constant $n \times n$ vector field (possibly perturbed) depending on the function $x_{t} ; g(x)=$ $\left[g_{1}(x) \ldots g_{m}(x)\right]$ is a non constant $n \times m$ control field, where $g_{i}(x), i \in\{1 . . m\}$ are sufficiently smooth vectors depending on $x(t)$;

$u \in \mathbb{R}^{m}$ is the control vector and $p$, representing all the neglected dynamics, is an exogenous signal. The initial condition $\phi$ is a piecewise continuous function. For vectors $e=\left(e_{1}, \ldots, e_{n}\right)^{T} \in \mathbb{R}^{n}$ and matrices $M=\left(m_{i j}\right) \in \mathbb{R}^{n \times n}$, the norm 2 is used $\|e\|=\sqrt{e^{T} e}$. For a symmetric matrix $M, M>0$ (resp. $M<0$ ) means that $M$ is positive (resp. negative) definite. $\lambda_{\min }(M)$ (resp. $\left.\lambda_{\max }(M)\right)$ stands for the largest (resp. the minus) eigenvalue of $M$.

For smooth $n$-vector fields, $f(x), g(x),[f, g](x) \triangleq$ $\frac{\partial g(x)}{\partial x} f(x)-\frac{\partial f(x)}{\partial x} g(x)$, (Lie bracket), generates a vector field and the $A d$ operator is defined by

$$
A d_{f}^{0} g(x) \triangleq g(x), \ldots, A d_{f}^{k} g(x) \triangleq\left[f, A d_{f}^{k-1} g\right](x) .
$$

Finally, $I_{r}$ denotes the set of the integers $\{1, \ldots, r\}$, and $\mathbb{N}^{*}$ is the set of strictly positive integers.

\section{assumptions}

A1) $\operatorname{rank}(g(x))=m$

Note that in the contrary case, it is possible to use 
a linear static feedback so to recover this assumption (see Appendix B).

A2) The perturbation satisfies the generalized matching conditions [Perruquetti et al., 1997] :

$$
p \in \operatorname{Vect}\left(A d_{g_{i}}^{k} g_{j}(x), i \in I_{m}, j \in I_{m}, k \in\{1 . . \infty\}\right) .
$$

A3) There exists a distribution $\Delta$ of dimension $m$ which satisfies $H 1, H 2, H 3$ with $d=m$. (see Appendix B)

\section{2 formulation of the problem}

Our goal in this section is to transform the system (1.1) into :

$$
\left\{\begin{aligned}
\dot{z}_{1} & =\sum_{i=1}^{r} h_{i}\left(z_{t}\right)\left(\begin{array}{cc}
{\left[\begin{array}{ll}
A_{i 11} & A_{i 12}
\end{array}\right] z(t)} \\
+\left[\begin{array}{ll}
A_{d i 11} & A_{d i 12}
\end{array}\right] z(t-\tau)
\end{array}\right) \\
\dot{z}_{2} & =f_{2}^{R}\left(z_{t}\right)+G_{2}^{R}(z) u(t)+p^{R}\left(z_{t}\right)
\end{aligned}\right.
$$

where $z, p^{R}$ represent the state and the perturbation in the new basis, $G_{2}^{R}(z)$ is a nonsingular matrix, and the $h_{i}\left(z_{t}\right), i \in I_{r}$ satisfy the convex sum property:

$$
\sum_{i=1}^{r} h_{i}\left(z_{t}\right)=1, h_{i}\left(z_{t}\right) \geq 0, i \in I_{r} .
$$

The transformation is achieved in two steps, as described in the following.

\subsection{Step One: Regular Form}

Lemma 1 Under assumptions $A 1, A 2, A 3$, the system (1.1) can be transformed into :

$$
\left\{\begin{array}{l}
\dot{z}_{1}=f_{1}^{R}\left(z_{1 t}, z_{2 t}\right), \\
\dot{z}_{2}=f_{2}^{R}\left(z_{1 t}, z_{2 t}\right)+G_{2}^{R}(z) u(t)+p^{R}\left(z_{t}\right),
\end{array}\right.
$$

where $G_{2}^{R}(z)$ is a nonsingular matrix, $z_{1} \in \mathbb{R}^{n-m}$, $z_{2} \in \mathbb{R}^{m}$

Proof. As vector field $g(x)$ does not depend on $x_{t}$ but on the instantaneous states $x(t)$, we can apply the procedure of the Appendix B to system (1.1).

\subsection{Step Two: Polytopic Formulation}

We suppose that the first subsystem can be factorized into the form :

$$
\left\{\begin{aligned}
\dot{z}_{1}= & {\left[\begin{array}{ll}
A_{11}\left(z_{t}\right) & A_{12}\left(z_{t}\right)
\end{array}\right] z(t) } \\
& +\left[\begin{array}{ll}
A_{d 11}\left(z_{t}\right) & A_{d 12}\left(z_{t}\right)
\end{array}\right] z(t-\tau), \\
\dot{z}_{2}= & f_{2}^{R}\left(z_{1 t}, z_{2 t}\right)+G_{2}^{R}(z) u(t)+p^{R}\left(z_{t}\right) .
\end{aligned}\right.
$$

We now assume that :

A4) Decomposition (2.4) holds with bounded matrices $A_{1 j}\left(z_{t}\right)$ and $A_{d 1 j}\left(z_{t}\right), j=1,2$.

A5) The perturbation $p^{R}\left(z_{t}\right)$ is bounded by a known functional $\Psi$ (possibly constant) as follows :

$$
\left\|p^{R}\right\|<\Psi\left(z_{t}\right)
$$

Under these assumptions, the first sub-system can be written under a polytopic formulation enhancing the bounds of the matrices $A_{1 j}\left(z_{t}\right)$ and $A_{d 1 j}\left(z_{t}\right)$ (see [Blanco \& Richard, 2000] for a complete proof). Indeed, there exists $r \in \mathbb{N}^{*}$, weighting functions $h_{i}\left(z_{t}\right)$, $i \in I_{r}$ and $r$ constant matrices $A_{i 1 k}, A_{d i 1 k}, i \in I_{r}$, $k \in I_{2}$ such that the first sub-system of (2.3) can be written as :

$$
\left\{\dot{z}_{1}=\sum_{i=1}^{r} h_{i}\left(z_{t}\right)\left(\begin{array}{l}
{\left[\begin{array}{ll}
A_{i 11} & A_{i 12}
\end{array}\right] z(t)} \\
+\left[\begin{array}{ll}
A_{d i 11} & A_{d i 12}
\end{array}\right] z(t-\tau)
\end{array}\right),\right.
$$

where the functions $h_{i}$ satisfy the convex sum property.

This part can be summarized in the following lemma :

Lemma 2 Under assumptions (A1-A5), the system (1.1) can be written as (2.1).

\section{Choice of the surface}

In order to design in sliding mode controller, let us choose a sliding surface

$$
s(z)=z_{2}+K z_{1} .
$$


System (2.4) is equivalent to :

$$
\left\{\begin{array}{l}
\dot{z}_{1}=\sum_{i=1}^{r} h_{i}\left(z_{t}\right)\left(\begin{array}{c}
E_{i} z_{1}(t)+E_{d i} z_{1}(t-\tau) \\
+A_{i 12} s(z)+A_{d i 12} s(t-\tau(t)),
\end{array}\right) \\
\dot{s}(z)=\xi\left(z_{t}\right)+G_{2}^{R}(z) u(t)+p^{R}\left(z_{t}\right),
\end{array}\right.
$$

with $E_{i}=A_{i 11}-A_{i 12} K, E_{d i}=A_{d i 11}-A_{d i 12} K$ and

$$
\begin{aligned}
& \left\{\begin{array}{l}
\xi\left(z_{t}\right)=\xi_{1}\left(z_{t}\right)+\xi_{2}\left(z_{t}\right), \\
\xi_{1}\left(z_{t}\right)=f_{2}^{R}\left(z_{1 t}, z_{2 t}\right) \\
\xi_{2}\left(z_{t}\right)=K \sum_{i=1}^{r} h_{i}(.)\left[\sum_{j=1}^{2} A_{j i 1} z_{j}(t)+A_{d j i 1} z_{j}(t-\tau)\right]
\end{array}\right. \\
& 4 \begin{array}{l}
\text { The case of a known and con- } \\
\text { stant delay }
\end{array}
\end{aligned}
$$

\subsection{Asymptotic stabilization}

Theorem 1 Let $\Lambda$ be a stable matrix and consider a $P>0, P=P^{T}$ such that $\Lambda^{T} P+P \Lambda=-I$. Under assumptions $(A 1-A 5)$, and if there exists two symmetric positive definite matrices $S, R$ and a matrix $W \in R^{m \times(n-m)}$ such that

$$
\left[\begin{array}{cc}
X_{i}+X_{i}^{T}+R & M_{i} \\
M_{i}^{T} & -R
\end{array}\right]<0, i \in I_{r}
$$

with $X_{i}=A_{i 11} S-A_{i 12} W, M_{i}=A_{d i 11} S-A_{d i 12} W$, then the control law

$$
\left\{\begin{array}{l}
u(t)=-G^{R}(z)^{-1}\left(\xi\left(z_{t}\right)-\Lambda s+m\left(z_{t}\right) \frac{P s}{\|P s\|}\right) \\
m\left(z_{t}\right)=m_{1}+\Psi\left(z_{t}\right) \\
m_{1}>0
\end{array}\right.
$$

makes $x=0$ asymptotically stable for (3.2) for all delays $\tau>0$.

The sliding surface (3.1) is then defined by $K=$ $W S^{-1}$.

Remark 1 It is possible to deal with the case of unknown and possibly perturbed matrices $A_{1 j}$ and $A_{d 1 j}$, assuming that they are included in known bounded domains. Then, non matching uncertainties can be integrated in our model (2.1). Simulation of this case is given in Section 6.

For such uncertainties, the numerical values of the functions $h_{i}$ are unknown, but it is possible to use an upperbound of the norm of $\xi$. Control law (4.2) is then transformed into:

$$
\left\{\begin{array}{l}
u(t)=-G^{R}(z)^{-1}\left(\xi_{1}\left(z_{t}\right)-\Lambda s+m\left(z_{t}\right) \frac{P s}{\|P s\|}\right) \\
m\left(z_{t}\right)=m_{1}+\Psi\left(z_{t}\right)+m_{2}\left(z_{t}\right) \\
m_{1}>0
\end{array}\right.
$$

$m_{2}\left(z_{t}\right)=\max _{i=1, r}\left(\left\|K\left\{\sum_{j=1}^{2} A_{i 1 j} z_{j}(t)+A_{d i 1 j} z_{j}(t-\tau)\right\}\right\|\right)$.

The proof of Theorem 1 will be achieved in three parts. We first prove the attractivity of the surface (with finite reaching time), then once in sliding motion (i.e. $s(z)=\dot{s}(z)=0$ ), we show the asymptotic stability of the reduced system. Finally, we ensure that the system in closed loop cannot become unbounded in finite time.

\section{Attractivity of the surface}

Lemma 3 Under assumptions $(A 1-A 5)$, if the LMIs (4.1) have a solution, the control (4.2) exists and makes the surface $s(z)=0$ stable and globally attractive in finite time.

Proof. Consider the function $V(t)=s^{T}(z) P s(z)$. Its derivative along the trajectories of (3.2) with (4.2) is

$\dot{V}(t)<-s^{T}(z) s(z)-2 s^{T}(z) P\left(p^{R}(z)+m\left(z_{t}\right) \frac{P s}{\|P s\|}\right)$,

$\dot{V}(t)<-s(z)^{T} s(z)-2 m_{1} \sqrt{\lambda_{\min }(P)} \sqrt{V}$,

$\dot{V}(t)<-2 m_{1} \sqrt{\lambda_{\min }(P)} \sqrt{V}$.

This last inequality is known to prove the finite time convergence of (3.2) towards the surface $s=0$ (see [Utkin, 1991]).

Stability of the reduced system 
This subsection involves a Lyapunov-Krasovskii approach.

If we suppose that the system reaches the sliding surface at time $t_{0}$, then once on the surface, the equations $s(z)=\dot{s}(z)=0$ hold and for $t>t_{0}+\tau$, the reduced system is driven by:

$$
\begin{aligned}
\dot{z}_{1} & =\sum_{i=1}^{r} h_{i}\left(z_{t}\right)\left(\left(A_{11}^{i}-A_{12}^{i} K\right) z_{1}(t)\right. \\
& \left.+\left(A_{d 11}^{i}-A_{d 12}^{i} K\right) z_{1}(t-\tau)\right)
\end{aligned}
$$

Lemma 4 The origin of the reduced system (4.5) is asymptotically stable if there exists $S>0, R>0$ and a matrix $W \in R^{m \times(n-m)}$ such that the $L M I$ (4.1) has a solution.

Proof. According to Theorem 3 (appendix A), the system :

$$
\dot{z}=\sum_{i=1}^{r} h_{i}\left(z_{t}\right)\left(A_{i} z_{1}(t)+A_{d i} z_{1}(t-\tau)\right)
$$

is asymptotically stable if there exists two symmetric positive definite matrices $P$ and $Q$ of $R^{(n-m) \times(n-m)}$ such that :

$$
\left[\begin{array}{cc}
A_{i}{ }^{T} P+P A_{i}+Q & P A_{d i} \\
A_{d i}{ }^{T} P & -Q
\end{array}\right]<0, i \in I_{r} .
$$

Replacing $A_{i}$ by $Z_{i}=\left(A_{i 11}-A_{i 12} K\right)$ and $A_{d i}$ by $N_{i}=A_{d i 11}-A_{d i 12} K$, we get

$$
\left[\begin{array}{cc}
Z_{i}^{T} P+P Z_{i}+Q & P N_{i} \\
N_{i}^{T} P & -Q
\end{array}\right]<0, i \in I_{r} .
$$

With a view to get LMIs, we pre- and post- multiply by $\left[\begin{array}{ll}S & 0 \\ 0 & S\end{array}\right]$, where $S=P^{-1}$ and get :

$$
\left[\begin{array}{cc}
S Z_{i}^{T}+Z_{i} S+R & N_{i} S \\
S N_{i}^{T} & -R
\end{array}\right]<0, i \in I_{r} .
$$

Finally, with the change of variables $W=K S$ and $R=S Q S$, (4.6) is equivalent to (4.1) which concludes the proof.

No escape in finite time
In this sub-section, we proove that the system (3.2) cannot become unbounded in finite time, for $t \in\left[0, t_{0}+\tau\right]$. It relies on the following LyapunovKrasovskii functional :

$$
\begin{gathered}
V\left(x_{t}\right)=V_{1}\left(x_{t}\right)+V_{2}\left(x_{t}\right), \\
V_{1}\left(x_{t}\right)=x(t)^{T} P x(t), \\
V_{2}\left(x_{t}\right)=\int_{t-\tau}^{t} x(\theta)^{T} S x(\theta) d \theta,
\end{gathered}
$$

which is also used in Appendix B.

The derivative of $V$ along the solutions of (3.2) leads to :

$$
\begin{aligned}
\dot{V}\left(z_{t}\right) & =X^{T}(t) M(.) X(t) \\
& +2 z_{1}^{T} P \sum_{i=1}^{r} h_{i}\left(z_{t}\right)\left(A_{i 12} s(t)+A_{d i 12} s(t-\tau)\right)
\end{aligned}
$$

where $X(t)^{T}=[x(t), x(t-\tau)], M()=.\sum_{i=1}^{r} h_{i}\left(x_{t}\right) M_{i}$, with $M_{i}=\left[\begin{array}{cc}G_{i}+S & B_{i}^{T} P \\ P B_{i} & -S\end{array}\right]$ and $G_{i}=A_{i}^{T} P+P A_{i}$.

Accrding to the proof of lemma $4, M$ is designed so that $M<0$.

Then, we get the following inequality :

$$
\dot{V}\left(z_{t}\right) \leq 2 z_{1}^{T} P \sum_{i=1}^{r} h_{i}\left(z_{t}\right)\left(A_{i 12} s(t)+A_{d i 12} s(t-\tau)\right)
$$

or

$$
\begin{aligned}
\dot{V} & \leq 2\left\|P \sum_{i=1}^{r} h_{i}\left(z_{t}\right) A_{i 12}\right\|\left\|z_{1}(t)\right\|\|s(z(t))\| \\
& +2\left\|P \sum_{i=1}^{r} h_{i}\left(z_{t}\right) A_{i d 12}\right\|\left\|z_{1}(t)\right\|\|s(z(t-\tau))\| .
\end{aligned}
$$

As $\sum_{i=1}^{r} h_{i}\left(z_{t}\right)=1$, one can find two positives constants $\delta$ and $\eta$ (independent of $z_{t}$ ) such that :

$$
\dot{V} \leq \delta\left\|z_{1}(t)\right\|\|s(z(t))\|+\eta\left\|z_{1}(t)\right\|\|s(z(t-\tau))\| .
$$


Futhermore, there exists a real $\mu>0$, such that $\left\|z_{1}(t)\right\| \leq \mu V_{1}^{\frac{1}{2}}$ and as $V_{1}<V$, we obtain :

$$
\dot{V} \leq \delta \mu V^{\frac{1}{2}}\|s(z(t))\|+\eta \mu V^{\frac{1}{2}}\|s(z(t-\tau))\| .
$$

According to Lemma $3, s$ is converging toward zero in finite time. Its norm can be bounded by a constant $s_{o}$. Then, we can bound the evolution of $V$ by a function $W$ such that :

$$
\dot{W}=(\delta+\eta) \mu s_{o} W^{\frac{1}{2}} .
$$

We can show easily that $W$ cannot escape to the infinite in finite time. Then the first sub-system, as the overall system, cannot escape in finite time.

\section{The case of a unknown, vary- ing but bounded delay}

\subsection{Asymptotic stabilization}

In this part, for the simplicity of the notations, we note $\tau=\tau(t)$.

We assume that the precise value of the delay is an unknown and continuous function of $t$, but bounded by $\tau_{\min }$ and $\tau_{\max }$ so that $\tau(t) \in\left[\tau_{\min } ; \tau_{\text {sup }}\right]$.

Theorem 2 Let $\Lambda$ be a stable matrix and a $P>0$, $P=P^{T}$ such that $\Lambda^{T} P+P \Lambda=-I$. Under assumptions $(A 1-A 5)$ and if there exists a symmetric positive definite matrix $S$ and a matrix $W \in R^{m \times(n-m)}$ such that

$$
\left[\begin{array}{cc}
X_{i}+X_{i}^{T}+S & M_{i} \\
M_{i}^{T} & -S
\end{array}\right]<0, i \in I_{r}
$$

then the control law

$$
\left\{\begin{array}{l}
u(t)=-G_{2}^{R}(z)^{-1}\left(-\Lambda s+m\left(z_{t}\right) \frac{P s}{\|P s\|}\right), \\
m\left(z_{t}\right)=m_{1}+\sup _{w \in\left[\tau_{\text {min }}, \tau_{\text {sup }}\right]}\left(\Psi\left(z_{t}(w)\right)\right) \\
+\sup _{w \in\left[\tau_{\text {min }}, \tau_{\text {sup }}\right]}\left\|\xi\left(z_{t}(w)\right)\right\| \\
m_{1}>0,
\end{array}\right.
$$

makes $x=0$ asymptotically stable for (3.2) for all delays $\tau>0$. The sliding surface (3.1) is then defined by $K=W S^{-1}$.
Remark 2 Similarly to the Krasovskii's approach, the knowledge of the functions $h_{i}$ is not necessary. Non matching uncertainties can then be involved. The control law considered is then :

$$
\left\{\begin{array}{l}
u(t)=-G^{R}(z)^{-1}\left(-\Lambda s+m\left(z_{t}\right) \frac{P s}{\|P s\|}\right), \\
m\left(z_{t}\right)=m_{1}+\sup _{w \in\left[\tau_{\text {min }}, \tau_{s u p}\right]}\left(\Psi\left(z_{t}(w)\right)\right)+ \\
\sup _{w \in\left[\tau_{\text {min }}, \tau_{\text {sup }}\right]}\left(\left\|\xi_{1}\left(z_{t}(w)\right)\right\|+m_{2}\left(z_{t}(w)\right),\right. \\
m_{1}>0,
\end{array}\right.
$$

$m_{2}\left(z_{t}(w)\right)=\max _{i=1, r}\left(\left\|\begin{array}{c}K\left(A_{i 11} z_{1}(t)+A_{d i 11} z_{1}(t-s)\right. \\ \left.+A_{i 12} z_{2}(t)+A_{d i 12} z_{2}(t-s)\right)\end{array}\right\|\right)$

The proof is achieved in three parts similarly to Krasovskii's case.

\section{Attractivity of the surface}

Lemma 5 Under assumptions $(A 1-A 5)$, if the LMIs (5.1) have a solution, the control (5.2) exists and makes the surface $s(z)=0$ stable and globally attractive in finite time.

Proof. Consider the functional $V(t)=$ $s^{T}(z) P s(z)$. Its derivative along the trajectories of (3.2) with $(5.2)$ is

$$
\begin{aligned}
\dot{V}(t) & <-s^{T}(z) s(z) \\
& -2 s^{T}(z) P\left(p^{R}(z)-\xi\left(z_{t}\right)+m\left(z_{t}\right) \frac{P s}{\|P s\|}\right), \\
\dot{V}(t) & <-s(z)^{T} s(z)-2 m_{1} \sqrt{\lambda_{\min }(P)} \sqrt{V} \\
\dot{V}(t) & <-2 m_{1} \sqrt{\lambda_{\min }(P)} \sqrt{V} .
\end{aligned}
$$

This last inequality is known to prove the finite time convergence of (3.2) towards the surface.

\section{Stability of the reduced system}

This subsection involves a Lyapunov-Razumikhin approach.

If we suppose that the system reach the sliding surface at time $t_{0}$, then, once on the surface, the equations $s(z)=\dot{s}(z)=0$ hold and for $t>t_{0}+\tau$, the system is driven by equation (3.2). 
Lemma 6 The reduced system (3.2) is asymptotically stable if there exists two symmetric definite positive matrices $S, R$ and a matrix $W \in R^{m \times(n-m)}$ such that LMIs (5.1) are verified.

Proof. Let us choose the function of Razumikhin as follows:

$$
V(z)=z_{1}^{T} P z_{1},
$$

where $P$ is a symmetric positive definite matrix $\epsilon$ $R^{(n-m) \times(n-m)}$.

Suppose there exists a class of functional solutions $\phi$ such that :

$$
\|V(\phi(t-s))\|<q\|V(\phi(t))\|, s \in\left[0, \tau_{\max }\right],
$$

where $q>1$. Then, the following inequality holds:

$$
\phi(t-s)^{T} P \phi(t-s)<q \phi(t)^{T} P \phi(t) .
$$

The reduced system along the solution $\phi_{t}$ is driven by the following equation :

$$
\dot{\phi}=\sum_{i=1}^{r} h_{i}\left(\phi_{t}\right)\left\{\begin{array}{c}
\left(A_{i 11}-A_{i 12} K\right) \phi(t) \\
+\left(A_{d i 11}-A_{d i 12} K\right) \phi(t-\tau)
\end{array}\right\} .
$$

Let us denote :

$$
\begin{aligned}
& A_{T S}=\sum_{i=1}^{r} h_{i}\left(\phi_{t}\right)\left(A_{i 11}-A_{i 12} K\right), \\
& \begin{aligned}
A_{d T S}= & \sum_{i=1}^{r} h_{i}\left(\phi_{t}\right)\left(A_{d i 11}-A_{d i 12} K\right) . \\
\dot{V}(\phi(t)) & =\phi(t)^{T}\left(A_{T S}^{T} P+P A_{T S}\right) \phi(t) \\
& +2 \phi(t)^{T} P A_{d T S} \phi(t-\tau) .
\end{aligned}
\end{aligned}
$$

Using the following inequality :

$$
\begin{aligned}
2 \phi(t)^{T} P A_{d T S} \phi(t-\tau) & \leq \phi(t)^{T} P A_{d T S} P^{-1} A_{d T S}^{T} P \phi(t) \\
& +\phi(t-\tau)^{T} P \phi(t-\tau),
\end{aligned}
$$

and the property (5.5), we finally get :

$\dot{V}(\phi(t)) \leq \phi(t)^{T}\left(\begin{array}{c}A_{T S}^{T} P+P A_{T S} \\ +P A_{d T S} P^{-1} A_{d T S}^{T} P+q P\end{array}\right) \phi(t)$.

Then, if there exists a real $q>1$ such that :

$$
\left[\begin{array}{cc}
Z_{i}^{T} P+P Z_{i}+q P & P N_{i} \\
N_{i}^{T} P & -P
\end{array}\right]<0, i \in I_{r},
$$

for $Z_{i}=A_{i 11}-A_{i 12} K, N_{i}=A_{d i 11}-A_{d i 12} K$, then the reduced system (3.2) is asymptotically stable.

By pre- and post-multiplying by $\left[\begin{array}{cc}S & 0 \\ 0 & S\end{array}\right]$, where $S=P^{-1}$ we get :

$$
\left[\begin{array}{cc}
S Z_{i}^{T}+Z_{i} S+q S & N_{i} S \\
S N_{i}^{T} & -S
\end{array}\right]<0, i \in I_{r} .
$$

Finally, with the change of variables $W=K S,(5.7)$ is equivalent to :

$$
h(q)=\left[\begin{array}{cc}
X_{i}+X_{i}^{T}+q S & M_{i} \\
M_{i}^{T} & -S
\end{array}\right]<0, i \in I_{r},
$$

By continuity of the functions $q \longmapsto h(q)$, if the LMIs (5.1) are satisfied, then there exists $q>1$ such that (5.8) is verified. The proof follows.

\section{No escape in finite time}

The idea of the proof is exactly the same as the Krasovovskii's approach, but relies on a LyapunovRazumikhin approach and will not be developped.

\section{Example}

Let us consider the following model with unknown (bounded) perturbations $\beta(t)$ and $f(t)$ :

$$
\begin{aligned}
\dot{x}(t)= & {\left[\begin{array}{ccc}
-3 & 2 & 1 \\
2 & 1+\sin \left(x_{3}(t)\right) & 1 \\
1 & \multicolumn{1}{c}{1} & \left(x_{2}(t)\right)^{2}+1
\end{array}\right] x(t) } \\
& +\left[\begin{array}{ccc}
0.5 & 0 & 0 \\
0 & 1 & 0.2 \\
-0.2 & -0.5 & 1
\end{array}\right] x(t-\tau) \\
+ & {\left[\begin{array}{l}
0 \\
0 \\
1
\end{array}\right] u(t)+\left[\begin{array}{c}
\beta(t) x_{1}(t) \\
-0.5 \beta(t) x_{1}(t-\tau) \\
f(t)
\end{array}\right], }
\end{aligned}
$$

with $0 \leq \beta(t) \leq 2$, and $f(t) \in[-1,1]$ is a disturbance function $(f(t)=\cos (100 t)$ in the simulations).

The various matrices used for transforming the original system into a polytopic formulation are : 


$$
\begin{aligned}
A_{i 12} & =\left[\begin{array}{l}
1 \\
1
\end{array}\right], A_{d i 12}=\left[\begin{array}{c}
0 \\
0.2
\end{array}\right], \\
A_{1 d 11} & =A_{3 d 11}=\left[\begin{array}{cc}
0.5 & 0 \\
0 & 1
\end{array}\right], \\
A_{2 d 11} & =A_{4 d 11}=\left[\begin{array}{ll}
0.5 & 0 \\
-1 & 1
\end{array}\right], \\
A_{111} & =\left[\begin{array}{cc}
-3 & 2 \\
2 & 1
\end{array}\right], A_{211}=\left[\begin{array}{cc}
-1 & 2 \\
2 & 1
\end{array}\right], \\
A_{311} & =\left[\begin{array}{cc}
-3 & 2 \\
2 & 0
\end{array}\right], A_{411}=\left[\begin{array}{cc}
-1 & 2 \\
2 & 0
\end{array}\right] .
\end{aligned}
$$

The initial function is $x(t)=[1,1,-1]^{T}$ for $t \in$ $[-\tau, 0]$.

In the simulation of Figure 1, we assume that the delay is known and constant. Since the system is in regular form, we can apply directly the method proposed in Section 4. The control (4.2) is applied using Theorem 1 : system (6.1) is then proved to be asymptotically stabilized for $\tau \geq 0$. By convex optimization based on the numerical resolution of the constraints (4.1), we find the coefficients of the surface :

$$
K=\left[\begin{array}{ll}
0.496 & 3.04
\end{array}\right] .
$$

The simulation are obtained using a $5^{\text {th }}$ order integration scheme with a step size of 0.001 and choosing $\Lambda=-1, m_{1}=1, \tau=0.8$. The surface is reached in approximatively $1 \mathrm{~s}$. Once on the surface, the reduced system tends towards the equilibrium point in $2.5 \mathrm{~s}$. Finally, the system is stabilized in $\simeq 3 s$ in spite of a delay $\tau=0.8 s$. We note there exists a chattering with an amplitude of 1 , which can be reduced by using a smooth approximation of the function $\frac{P s}{\|P s\|}$.

Remark 3 Note that the sliding mode controllers proposed in [Choi, 1999, Shyu \& Yan, 1993] cannot stabilize system (6.1) since it is nonlinear. Moreover, the classical state feedback [Dambrine et al., 1995, Niculescu, 1998, Yu \& Chu, 1999] cannot reject the perturbation since $f$ is not vanishing. Controllers based on nonlinear models over rings [Moog et al., 2000] may achieve the disturbance rejection but needs to assume that the delay is exactly known and constant which is not the case.

\section{Conclusion}

In this paper, the problem of the robust stabilization of nonlinear time delay system has been studied by designing a sliding mode controller. Both constant known delays and unknown varying delays have been investigated. All the required computations required are expressed in term of a convex optimization problem with LMI constraints, allowing for the use of efficient solvers.

A stabilization example of a nonlinear delay system with a constant or varying known delay and nonmatching uncertainties emphasized the interest of the proposed method.

\section{Appendix A}

In this part, we derive sufficient conditions for the system

$$
\dot{x}(t)=\sum_{i=1}^{r} h_{i}\left(x_{t}\right)\left(A_{i} x(t)+B_{i} x(t-\tau)\right),
$$

to be asymptotically stable [Blanco \& Richard, 2000] for more details).

Theorem 3 Consider system (8.1). If there exists two symmetric positive definite matrices $P$ and $S$ of $R^{n \times n}$ such that:

$$
\left[\begin{array}{cc}
A_{i}^{T} P+P A_{i}+S & B_{i}^{T} P \\
P B_{i} & -S
\end{array}\right]<0
$$

then the equilibrium $x=0$ of the system (8.1) is globally asymptotically stable for all delays $\tau>0$.

Proof. Consider the following LyapunovKrasovkii functional :

$$
\begin{gathered}
V\left(x_{t}\right)=V_{1}\left(x_{t}\right)+V_{2}\left(x_{t}\right), \\
V_{1}\left(x_{t}\right)=x(t)^{T} P x(t), \\
V_{2}\left(x_{t}\right)=\int_{t-\tau}^{t} x(\theta)^{T} S x(\theta) d \theta,
\end{gathered}
$$


with $P=P^{T}, P>0$ and $S=S^{T}, S>0$. We have $\beta_{1}\|x(t)\|^{2} \leq V\left(x_{t}\right) \leq \beta_{2}\left\|x_{t}\right\|_{c}^{2}$, with $\beta_{1}=\lambda_{\min }(P)$, $\beta_{2}=\lambda_{\max }(P)+\tau \lambda_{\max }(S)$. Let us now calculate the derivative of $V\left(x_{t}\right)$ along the trajectory of the system (8.1):

$$
\begin{aligned}
\dot{V}_{2}\left(x_{t}\right)= & x(t)^{T} S x(t)-x(t-\tau)^{T} S x(t-\tau), \\
\dot{V}_{1}\left(x_{t}\right)= & \dot{x}(t)^{T} P x(t)+x(t)^{T} P \dot{x}(t), \\
= & \left\{\begin{array}{c}
x(t)^{T}\left[\sum_{i=1}^{r} h_{i}\left(x_{t}\right)\left(A_{i}^{T} P+P A_{i}\right)\right] x(t) \\
+2 x(t-\tau)^{T}\left[\sum_{i=1}^{r} h_{i}\left(x_{t}\right) B_{i}^{T} P\right] x(t) \\
+2 x(t)^{T}\left[\sum_{i=1}^{r} h_{i}\left(x_{t}\right) P B_{i}\right] x(t-\tau) .
\end{array}\right.
\end{aligned}
$$

Defining $X(t)^{T}=[x(t), x(t-\tau)]$ leads to $\dot{V}\left(x_{t}\right)=X^{T}(t) M() X.(t)$ with

$$
\left\{\begin{array}{l}
M(.)=\sum_{i=1}^{r} h_{i}\left(x_{t}\right) M_{i} \\
M_{i}=\left[\begin{array}{cc}
G_{i}+S & B_{i}^{T} P \\
P B_{i} & -S
\end{array}\right] \\
G_{i}=A_{i}^{T} P+P A_{i} .
\end{array}\right.
$$

A sufficient condition for $\dot{V}\left(x_{t}\right)$ to be negative definite is

$$
M(.)<0 .
$$

Since $h_{i}\left(x_{t}\right) \geq 0, \forall i \in I_{r}$ and $\exists i_{0} \in I_{r}: h_{i_{0}}\left(x_{t}\right)>0$, (8.4) holds if $M_{i}<0, \forall i \in I_{r}$. Consequently, by virtue of Krasovskii theorem, if there exists $P$ and $S$ such that (8.3) holds, system (8.1) is globally asymptotically stable for all delays $\tau>0$.

\section{Appendix B : Generalized regular form}

In this section, we consider the following MIMO system of the form :

$$
\dot{x}=f(x)+g(x) u(t)+p(x)
$$

where $p$ is an additive perturbation, $f$ is a sufficiently smooth differential vector field, $g(x)=\left(\begin{array}{lll}g_{1}(x) & \ldots & g_{m}(x)\end{array}\right)$ is a $n \times m$ matrix where $g_{i}(i \in\{1 . . m\})$ are sufficiently smooth vectors fields. In the following, we investigate the conditions under whcih the system (9.1) can be transformed into the form :

$$
\left\{\begin{array}{l}
\dot{z}_{1}=f_{1}^{R}\left(z_{1}, z_{2}\right)+G^{R}\left(z_{1}, z_{2}\right) u+p^{R}(z), \\
\dot{z}_{2}=f_{2}^{R}\left(z_{1}, z_{2}\right) \\
z_{1} \in R^{d}, z_{2} \in R^{n-d} .
\end{array}\right.
$$

The results presented here have been developped in [Lukyanov \& Utkin, 1981] in the unperturbed case and in [Perruquetti et al., 1997] for the general case.

\subsection{Rank of the input matrix}

In [Lukyanov \& Utkin, 1981], it is supposed that $g(x)$ is full-rank matrix. When this assumption is not true, it is possible to use a linear static feedback in order to recover Lukyanov's assumption.

Theorem 4 If $\left.\operatorname{rank}\left(g\left(x_{0}\right)\right)\right)=r$ then there exists a static feedback $u=W(x)\left(v^{T}, 0,0, \ldots, 0\right)^{T}, v \in \mathbb{R}^{r}$, with $v$ the new input and $W$ a nonsingular in a neighbourhood of $x_{0}$ such that

$$
\begin{aligned}
& g(x) W(x)=\left(\begin{array}{ccccccc}
\overbrace{X} & 0 & \cdots & 0 & 0 & \cdots & 0 \\
\vdots & \ddots & \ddots & \vdots & \vdots & & \vdots \\
\vdots & & \ddots & 0 & \vdots & & \vdots \\
X & \cdots & \cdots & X & 0 & \cdots & 0 \\
X & \cdots & \cdots & X & 0 & \cdots & 0 \\
\vdots & & & \vdots & \vdots & & \vdots \\
X & \cdots & \cdots & X & 0 & \cdots & 0
\end{array}\right) \\
& =(\overbrace{g^{\prime}(x)}^{r}{ }_{0}^{m-r})
\end{aligned}
$$

If $\left.\operatorname{rank}\left(g\left(x_{0}\right)\right)\right)=r<m$, the new system is written as

$$
\dot{x}=f(x)+g^{\prime}(x) v+p(x),
$$


where $v \in \mathbb{R}^{r}$ is the new input and $g^{\prime}(x)$ is a full rank $n \times r$ matrix.

For simplicity, it is now possible, without loss of generality, to consider that $\left.\operatorname{rank}\left(g\left(x_{0}\right)\right)\right)=m$.

The problem is to find a diffeomorphic state space transformation $z=\phi(x)$ changing (9.1) into (9.2).

\subsection{Obtention of the generalized regu- lar form in the unperturbed case.}

The given results are local, but when asumption $H 1$ ) (see theorem statements) holds everywhere in the state space, then the diffeomorphism is global and so are the results.

Theorem 5 Let $\Delta$ be a distribution such that:

H1) $\Delta$ is nonsingular at $x_{0}$ (i.e. of constant dimension $\operatorname{dim} \Delta=d_{\Delta} \leq n$ ),

HQ) $\Delta$ is involutive (i.e completly integrable):

$$
\forall \tau_{1} \in \Delta, \forall \tau_{2} \in \Delta:\left[\tau_{1}, \tau_{2}\right] \in \Delta
$$

H3) $\operatorname{span}\left\{g_{1}(x), \ldots, g_{m}(x)\right\} \subset \Delta$,

Then there exist a neighbourhood $\mathcal{V}\left(x_{0}\right)$ of $x_{0}$ and a local diffeomorphism $z=\phi(x)$ defined on $\mathcal{V}\left(x_{0}\right)$, such that (9.1) is transformed into (9.2) with $d=d_{\Delta} \leq n$. Moreover, if $d_{\Delta}<n$, then the conclusion holds for $n \geq d \geq d_{\Delta}$.

Proof (sketch): Under assumptions $H 1-H 3$, one can find $\left(n-d_{\Delta}\right)$ real valued functions $\lambda_{i}$ spanning the anihilator of $\Delta$ (Frobenius Theorem), selecting $\phi_{d+i} \triangleq \lambda_{i}$ and completing the basis leads to the result.

Remark 4 An algorithm for construction of a such distribution $\Delta$ can be found in [Perruquetti et al., 1997].

\subsection{Perturbed case.}

Theorem 6 [Perruquetti et al., 1997] Suppose that:

$$
\text { Ho) } p \quad \in \quad \Delta_{G} \quad=
$$
$\operatorname{span}\left\{A d_{g_{i}}^{k} g_{j}(x): i \in\{1 . . m\}, j \in\{1 . . m\}, k \in\{0 . . \infty\}\right\}$
$\left.H^{\prime} 0\right) \Delta_{G}$ is nonsingular in $x_{0}$ (i.e. of constant dimension $\operatorname{dim} \Delta_{G}=d_{\Delta_{G}} \leq n$ ).

Then, there exist a neighbourhood $\mathcal{V}\left(x_{0}\right)$ of $x_{0}$ and a local diffeomorphism $z=\phi(x)$ defined on $\mathcal{V}\left(x_{0}\right)$, such that (9.1) is transformed into (9.2) with $d=$ $d_{\Delta_{G}}$.

Proof: As $p \in \Delta_{G}, \forall \omega^{*} \in \Delta_{G}^{\perp}:<\omega^{*}, p>=0$, and using local diffeomorphism $\phi(x)$ defined in Theorem 15 , we obtain the result.

Remark 5 If $\Delta_{G}=$ vect $\left\{g_{1}(x), \ldots, g_{m}(x)\right\}$ is involutive, then assumption $\mathrm{HO}$ ) is the classical "matching condition" [Utkin, 1991]. 

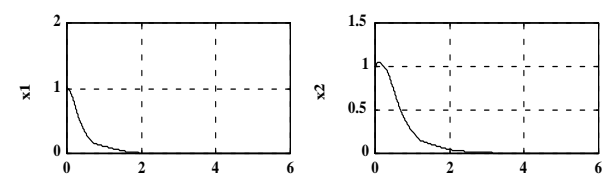

$\ddot{x}$
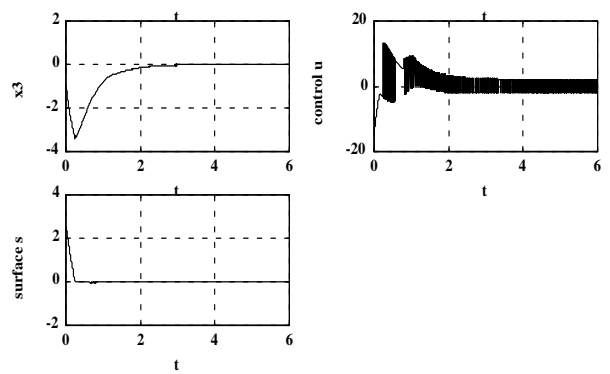

Figure 1: Simulation of (6.1), (4.2) with $m_{1}=1, \Lambda=$ $-1, \tau=0.8$

\section{References}

[Blanco \& Richard, 2000] BLAnco, Y., \& RichARD, J.P. 2000. Stability analysis of uncertain nonlinear systems with delay using takagi-sugeno fuzzy representations. In: Proc. LTDS'00, ancône.

[Bonnet et al., 1998] Bonnet, C., Partington, J.R., \& Sorine, M. 1998 (July). Robust control and tracking in $l_{\infty}$ of delay systems equipped with a relay sensor. In: Proc. 8th. IFAC conf. large scale systems LSS'98.

[Choi, 1999] Chог, H.H. 1999. An lmi approach to sliding mode control design for a class of uncertain time-delay systems. In: Proc. ECC'99.

[Conte \& Perdon, 1995] Conte, G., \& Perdon, A.M. 1995. The disturbance decoupling problem for systems over a ring. Siam $j$. control and optimization, $33(3)$.

[Dambrine et al., 1995] DAmbRIne, M., RICHARD, J.P., \& Borne, P. 1995. Feedback control of time-delay systems with bounded control and state. Mathematical problems in engineering, $\mathbf{1}$, 77-87. (first issue).
[Dambrine et al., 1998] Dambrine, M., GouaisBaut, F., Perruquetti, W., \& Richard, J.-P. 1998. Robustness of sliding mode control under delays effects: a case study. Pages 817-821 of: Proc. 2nd IEEE-IMACS CESA'98, comput. eng. in systems applications,

[Germani et al., 1996] Germani, A., Manes, C., \& PePE, P. 1996. Linearization of input-output mapping for nonlinear delay systems via static state feedback. Pages 599-602 (vol. 1) of: IEEEIMACS conf. on computational engineering in syst. applications CESA'96.

[Gopalsamy, 1992] Gopalsamy, K. 1992. Stability and oscillations in delay differential equations of population dynamics. Mathematics and Applications, vol. 74. Kluwer Acad.

[Gouaisbaut et al., 1999] Gounisbaut, F., PerruQuetTi, W., \& Richard, J.P. 1999. A sliding mode control for linear systems with input and state delays. In: Proc. 38th IEEE CDC'99.

[Gouaisbaut et al., 2001] GouaisBaut, F, Blanco, Y., \& Richard, J.P. 2001. Robust control of nonlinear time delay system: A sliding mode control design. In: Proc. 1th IFAC symposium NOLCOS'01.

[Goubet.-Bartholoméüs et al., 1997] Goubet .Bartholoméüs, A., Dambrine, M., \& RichaRd, J.P. 1997. Stability of perturbed systems with time-varying delay. Systems and control letters, 31(3), 155-163.

[Kolmanovskii \& Myshkis, 1999] KolmanovskiI, V.B., \& Myshkis, A. 1999. Introduction to the theory and applications of functional differential equations. Dordrecht: Kluwer Acad.

[Kolmanovskii et al., 1999] Kolmanovskiı, V.B., Niculescu, S.I., \& Richard, J.P. 1999. On the liapunov-krasovskii functionals for stability analysis of linear delay systems. Int. j. control, $\mathbf{7 2 ( 4 )}$, $374-384$.

[Koshkouei \& Zinober, 1996] Koshkouei, A.J., \& Zinober, A.S.I. 1996. Sliding mode time-delay 
systems. Pages 97-101 of: Proc. international workshop on VSS.

[Li \& De Souza, 1996] LI, X., \& De SouzA, C.E. 1996. Robust stabilization and $\mathrm{H}_{\infty}$ control of uncertain linear time-delay systems. Pages 113-118 of: Proc. 13th ifac world congress. Vol. H.

[Li \& Decarlo, 2003] Li, X, \& Decarlo, R. A. 2003. Robust sliding mode control of uncertain time delay systems. Int. journal of control, 76(13), 1296-1305.

[Lukyanov \& Utkin, 1981] Lukyanov, A.G., \& UTKIN, V.I. 1981. Methods of reducing equations of dynamics systems to regular form. Automat. remote control, $\mathbf{4 2}, 413-420$.

[Luo \& De la Sen, 1992] Luo, N., \& DE LA SEN, M. 1992. State feedback sliding mode controls of a class of time-delay systems. Pages 894-895 of: Acc'92 (american control conf.).

[Moog et al., 2000] Moog, C.H., CAStro Linares, R., Velasco Villa, M., \& Marquez Martinez, L.A. 2000. The disturbance decoupling problem for time-delay nonlinear systems. Ieee trans. aut. control, 45(5), 572-575.

[Nguang, 1998] NguANG, S.K. 1998 (December). Robust $\mathbf{H}_{\infty}$ control of a class of nonlinear systems with delayed state and control: A LMI approach. Pages 2384-2389 of: Proc. 37th IEEE CDC'98.

[Niculescu, 1998] Niculescu, S.I. 1998. H $_{\infty}$ memoryless control with an $\alpha$-stability constraint for time delays systems: an lmi approach. Ieee trans. aut. control, 43(5), 739-743.

[Niculescu et al., 1998] Niculescu, S.I., De Souza, C.E., Dugard, L., \& Dion, J.M. 1998. Robust exponential stability of uncertain systems with time-varying delays. Ieee trans. aut. control, 43(5), 743-748.

[Perruquetti et al., 1997] Perruquetti, W., Richard, J.P., \& Borne, P. 1997. A generalized regular form for sliding mode stabilization of mimo systems. In: Proc. 36th IEEE CDC'97.
[Richard, 2003] RichaRd, J.P. 2003. Time delay systems: An overview of some recent advances and open problems. Automatica, 39, 1667-1694.

[Shyu \& Yan, 1993] Shyu, K.K., \& Yan, J.J. 1993. Robust stability of uncertain time-delay systems and its stabilization by variable structure control. Int. $j$. of control, 57, 237-246.

[Sira-Ramirez \& Angulo-Nunez, 1998] SiRARamirez, H., \& Angulo-Nunez, M.I. 1998 (December). On the passivity based regulation of a class of delay differential systems. Pages 297-298 of: 37th ieee cdc'98 (conf. on decision 8 control).

[Takagi \& Sugeno, 1985] Takagi, T., \& Sugeno, M. 1985. Fuzzy identification of systems and its applications to modelling and control. Ieee trans. on s.m.c., 15, 116-132.

[Tanaka et al., 1998] TAnAKA, K., IkedA, T., \& WANG, H. 1998. Fuzzy regulators and fuzzy observers design: Relaxed stability conditions and LMI-based design. Ieee trans. on fuzzy systems, $6(2), 14-23$.

[Utkin, 1991] UtKin, V.I. 1991. Sliding modes in control optimization. CCES Springer-Verlag.

[Yu \& Chu, 1999] Yu, L., \& Chu, J. 1999. An LMI approach to guarenteed cost control of linear uncertain time-delay systems. Automatica, 35, 11551159 . 